\title{
Observational aspects of asteroseismology
}

\author{
G. Handler \\ Institut für Astronomie, Türkenschanzstr. 17, 1180 Vienna, Austria
}

\begin{abstract}
We review past and present efforts to perform asteroseismic studies of the distant stars from an observational viewpoint. We discuss the challenges, problems and successes for the different kinds of pulsators that seem suitable for asteroseismology and we outline some paths along which the understanding of the interior structure of these stars could progress.
\end{abstract}

\section{Introduction}

Asteroseismology is the study of the interior of (non-radially) pulsating stars by means of their normal mode spectrum. This technique is analogous to the determination of the Earth's inner structure using earthquakes: we use stellar pulsation modes as seismic waves.

A pulsation mode is an individual stellar oscillation. The latter come in many different flavours, and it is impossible to give a complete overview in the present limited space; we refer the reader to the excellent monograph by Unno et al. (1989) for more detailed information. For the purpose of this article, it is most important to know that there are two main groups of pulsation modes, the pressure $(\mathrm{p})$ and the gravity $(\mathrm{g})$ modes. These modes are classified after the force that restores the stellar equilibrium shape following the motion caused by pulsation, i.e. it is either pressure or buoyancy.

Pulsation modes are also classified after the shape of the distortions they create on the stellar surface and interior. In general, the stellar surface is separated into expanding and receding as well as heating or cooling areas. The shape of these distortions can be quantified with spherical harmonics; an example of these is shown in Fig. 1.

Obviously, between the expanding and receding areas, no motion takes place. The lines along which this is the case are called the nodal lines. The number and direction of these nodal lines are used for the classification of the pulsation modes. The total number of nodal lines on the stellar surface is called the spherical degree $\ell$, which must in all cases be larger or equal to zero. The number of nodal lines that are intersected when travelling around the stellar equator is called $m$, the azimuthal order. Pulsation modes with $m \neq 0$ are travelling waves, and as they can run either with or against the rotation of the star, $m$ can lie in the interval $-\ell, \ell$. Finally, the number of nodal lines in the stellar interior is called the radial overtone $k$ (or sometimes $n$ ). With these three pulsational quantum numbers, any given pulsation mode can be described (to first order).

Asteroseismology takes advantage of the fact that some stars can oscillate in many of these radial and/or non-radial modes simultaneously. This is important, as each pulsation mode carries information about the region in which it propagates, its pulsation cavity. Every single pulsation mode has a different cavity, and its oscillation frequency is governed by the physical conditions in its cavity. 


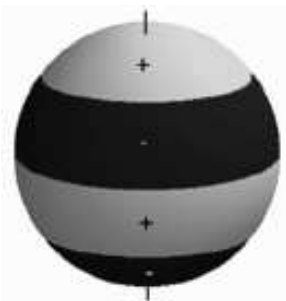

$|m|=0$

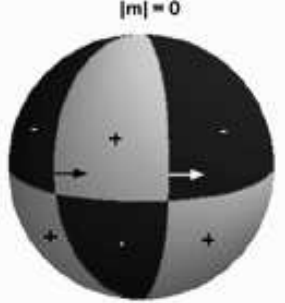

$|\mathrm{m}|=2$

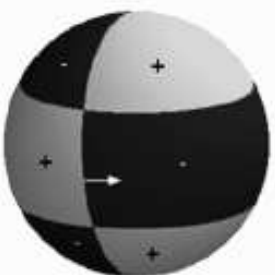

$|m|=1$

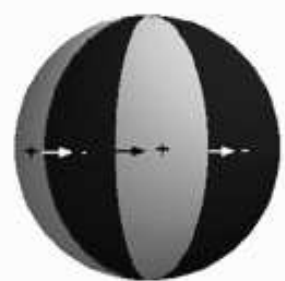

$|\mathrm{m}|=t=3$

Figure 1: Schematic description of the surface distortions produced by pulsation modes with a spherical degree $\ell=3$. Whilst the light-coloured areas of the star are moving outward, the darker parts move inward, and vice versa. Between those areas no motion takes place; these are the node lines. The total number of node lines on the surface is $\ell$, the number of node lines intersecting the equator is $m$. Graphics courtesy by W. Zima.

By measuring the oscillation frequencies of pulsating stars and by trying to reproduce them with stellar models, one thus can refine interior structure models of the stars. The prerequisite for successful seismic modelling, however, is that the observer provides the largest possible number of intrinsic pulsation frequencies with a correct identification of all the underlying modes to the theorist.

There are reasons beyond an improved understanding of stellar structure to engage in asteroseismology. For instance, some of the most massive pulsating stars will become Type II Supernovae in the future. Supernova explosions are mostly responsible for the enrichment of the interstellar medium. If we know the interior structure of an immediate supernova progenitor, we will therefore be able to constrain the chemical evolution of galaxies. Asteroseismology of pulsating white dwarf stars teaches us about nuclear reaction rates, neutrino physics and the history of the object's evolution on the Asymptotic Giant Branch. It is therefore clear that asteroseismology has a large impact on astrophysics in general.

\section{Success stories and challenges}

Of course, the greatest successes of asteroseismology concern the star closest to us, the Sun. The interior structure of the Sun is known to fine detail (e.g., see Christensen-Dalsgaard 2002 for an extensive review) as millions of pulsation modes can be used for seismic analyses. Because the solar surface can be resolved in two dimensions, even local asteroseismology (e.g., dealing with subsurface and meridional flows) can be carried out. However, the recent revisions in the solar elemental abundances suggest that our present solar model may need some revision. We refer to the contributions by Grevesse et al. and Montalban et al. to the present proceedings for more information. 


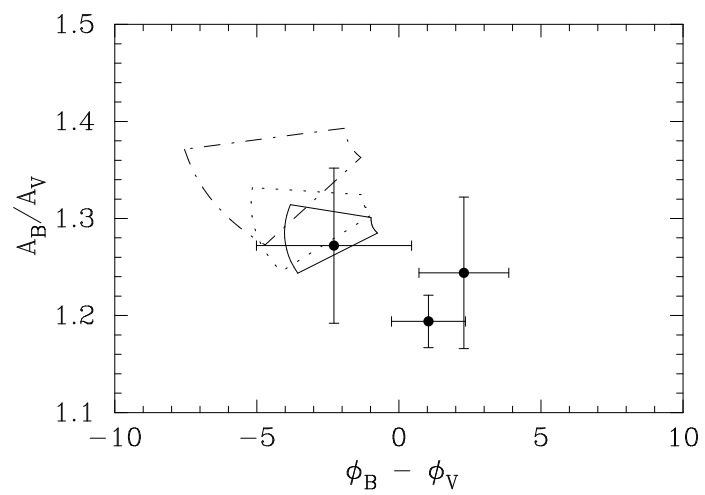

Figure 2: Amplitude ratios and phase shifts for the three strongest modes of $X X$ Pyx compared with theoretically predicted values. Boundaries for the $\ell=0$ area of interest are shown with full lines, those for the $\ell=1$ area with dotted lines and those for $\ell=2$ with dash-dotted lines. Taken from Handler et al. (2000).

If we compare the Sun and the distant stars, two major problems for asteroseismology become immediately obvious: we have to work with integrated light as we cannot (sufficiently) resolve stellar surfaces and we have much less light available. This means that we will only have a restricted range of modes, depending on their $\ell$ values, available for analysis, and that the signal-to-noise of the observations is poorer. Nevertheless, asteroseismology of some pulsating white dwarf stars (e.g., see Winget et al. 1991, 1994) was quite successful, resulting in exact determinations of masses, rotation periods, luminosities, and chemical element layer masses, as well as in estimates of magnetic field strengths.

Why was this possible? Pulsating white dwarfs oscillate in g modes of high radial order. Asymptotic theory then predicts that consecutive overtones are equally spaced in period (Tassoul 1980). The pulsation frequencies of these stars were arranged in clear patterns, and the underlying pulsation modes were identified by just examining the stars' frequency spectra.

Unfortunately, this method does not work for all pulsating stars. Even in cases where a sufficient number of pulsation modes were detected that patterns within their frequencies should become visible, they escaped detection (e.g., see Handler et al. 2000). A second problem that is common in the analysis of asteroseismic data is that only a small percentage of the theoretically predicted number of pulsation modes is actually observed, and that it is not clear which particular set of modes the star chooses to excite to measurable amplitude.

Consequently, we require additional observational methods that allow an identification of the stellar pulsation modes. This is facilitated by the interplay of the different surface distortions and the changes in gravity and temperature caused by a given mode combined with limb darkening effects. Therefore, amplitude ratios or phase shifts between different photometric passbands, also in combination with radial velocity data, can reveal the pulsation mode (Dziembowski 1977). The line profiles of an oscillating star will also reflect the pulsations (Ledoux 1951) and can thus be used to identify the mode.

However, are such mode identification methods really trustworthy? To illustrate this concern, Fig. 2 shows a comparison of theoretically predicted and observed amplitude ratios and phase shifts for the $\delta$ Scuti star XX Pyxidis. The observations for one of the modes do not fit the theoretical predictions at all, another one has a marginally consistent amplitude ratio but inconsistent phase shift and the third is consistent in both parameters; due to the results of the other two modes this one can however not be trusted either. 
We conclude that unless the mode identifications from any individual technique are unambiguous, extreme care must be taken when applying such methods. If possible, cross-checks between different techniques must be made, but it must also be checked if they are indeed (mostly) independent. We refer to the discussion by Balona (2000) who showed that the agreement of the mode identifications derived from one photometric and one spectroscopic technique were not due to their reliability, but resulted from the two methods being basically sensitive to the same quantities.

For planning an observational project it is thus suggested that it be set out that mode identification methods can be applied, but their limitations must be kept in mind. Another observational challenge for asteroseismology is that pulsational signals of extremely low amplitude (less than $1 \mathrm{mmag}$ or a few $\mathrm{cm} / \mathrm{s}$ ) need to be detected reliably, requiring the acquisition of large amounts of high-quality measurements. Finally, the observer should be careful to measure stars that theory can treat within its current limitations; close interaction between observers and theorists is therefore required. In fact, the subfields of asteroseismology where the first successes were obtained, benefited from just this interaction.

\section{The state of the art}

There are several classes of pulsating star that seem accessible to asteroseismic methods. An overview of the location of pulsating stars in the HR diagram is given in Fig. 3 . In the following, we comment on the status of the asteroseismic investigations for pulsating stars other than the Sun.

\subsection{Solar-like oscillators}

Stochastically excited high radial order $\mathrm{p}$ modes have now convincingly been detected in several solar-like stars from high-precision radial velocity measurements (e.g., see Butler et al. 2004), with spherical degrees $\ell$ up to 3 , and up to 42 detected modes (Bedding et al. 2004). As in the Sun, the oscillations seem to have short (compared to the time span of the observations) lifetimes, which would decrease the precision of the frequency determinations. Although detailed asteroseismic modelling has so far not been done for these objects, we believe that it is only a question of time that the first seismic models become available.

\subsection{Rapidly oscillating Ap stars}

The rapidly oscillating $A p(r o A p)$ stars are cool, magnetic, chemically peculiar A-type stars that pulsate with periods in the range of $5.6-21 \mathrm{~min}$ and with Johnson $B$ semi-amplitudes $<0.008 \mathrm{mag}$. These oscillations are caused by global $\mathrm{p}$-mode pulsations of low spherical degree $(\ell<3)$ and high radial overtones.

Photometric observations by Whole Earth Telescope network resulted in the detection of eight independent pulsation modes of the roAp star HR 1217 (Kurtz et al. 2005). The precision of the amplitude determinations for the individual frequencies is a stunning $14 \mu \mathrm{mag}$, making this the most precise ground-based photometric measurements obtained so far. The frequency spacings within these modes are consistent with theoretical predictions (e.g. Cunha 2001) but detailed seismic modelling has not been possible so far. The MOST asteroseismic satellite (www.astro.ubc.ca/MOST/) has also observed HR 1217, but the data have not been published at the time of this writing.

Spectroscopic observations of roAp stars, however, are even more revealing than photometry. The reason is that the vertical wavelength of the pulsation modes is shorter than or about the same order of the layer thicknesses of the chemical elements in the highly stratified atmospheres of these stars (Ryabchikova et al. 2002), and therefore the radial velocity amplitudes of the oscillations change with line depth and from one chemical species to the next. 


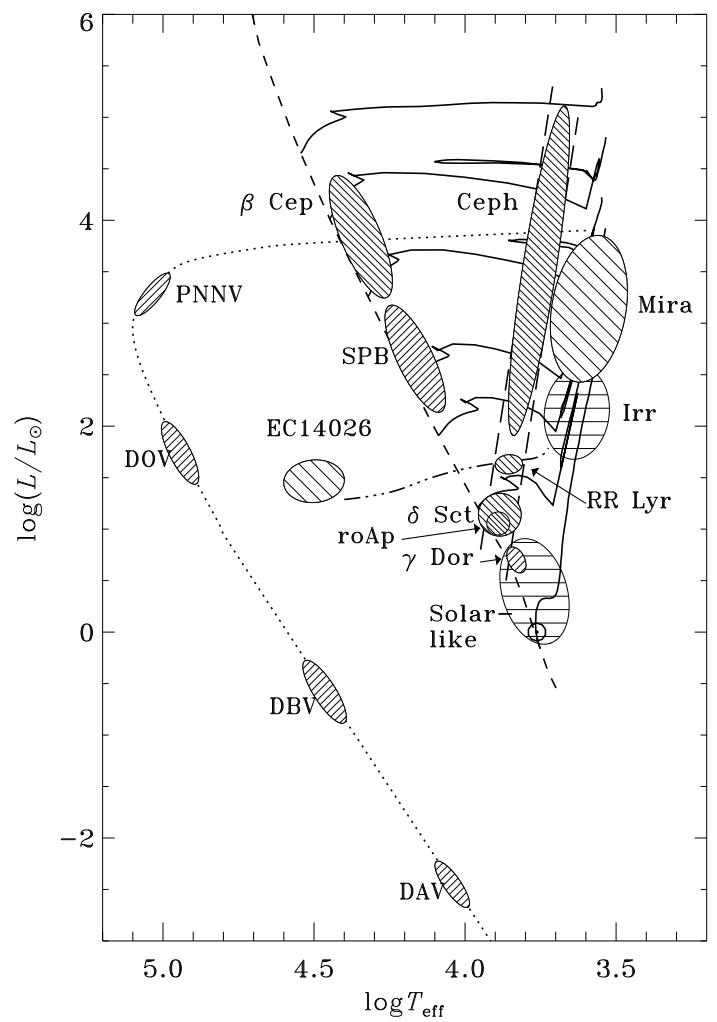

Figure 3: Theoretical HR diagram with many classes of pulsating star indicated. The dashed line is the theoretical main sequence, the dotted line the white dwarf cooling track and some evolutionary tracks are also indicated. Used with permission from J. Christensen-Dalsgaard.

For instance, the lines of Nd II and Nd III in the roAp star 33 Lib were shown to form at different sides of a pulsational node (Kurtz, Elkin \& Mathys 2005). It has also been concluded that the magnetoacoustic boundary layer in this star can be probed with the Nd III line data. Consequently, seismic analyses of the roAp stars are not yet able to probe their deep interior structure, but are highly capable of testing our understanding of their atmospheres.

\subsection{Delta Scuti stars}

These objects are single and multimode radial and nonradial pulsators that oscillate with periods between 20 minutes and 8 hours. They have spectral types of $A$ and $F$ and located in the classical instability strip in the HR diagram near the main sequence, but there is also a large population of subgiants and giants among the $\delta$ Scuti stars. The pulsation modes are $\mathrm{p}$ (and possibly $\mathrm{g}$ ) modes of low radial order.

Ground-based photometric observations of the $\delta$ Scuti stars reveal more and more pulsation 
frequencies, if persistently carried out. Breger et al. (2005) detected 67 independent mode frequencies for the star FG Virginis. This seems sufficient for seismic modelling, especially since firm identifications for several of the modes have been made (Zima, these proceedings). However, it needs to be kept in mind that at the low photometric amplitudes of most of the detected signals, modes with high spherical degree may be present (Daszyńska-Daszkiewicz et al. 2005) and could effectively mask the frequency patterns of the low-degree modes.

Another reason for optimism concerning seismology of $\delta$ Scuti stars is due to pre-main sequence pulsators. These stars are structurally simpler and should thus show simpler frequency spectra as main-sequence stars, facilitating mode identification. Indeed, more than a dozen modes were already discovered for some PMS pulsators (Kallinger, private communication).

Finally, it also seems that some mode identification methods become more reliable for the $\delta$ Scuti stars. Daszyńska-Daszkiewicz et al. (2003) show how photometric observations in at least three passbands can not only be used to identify pulsation modes, but also to obtain constraints on the theoretical treatment of surface convection for these stars.

\subsection{Gamma Doradus stars}

In the HR diagram, the $\gamma$ Doradus stars are located in the same region as the $\delta$ Scuti and roAp stars, but they are quite different in their pulsational behaviour. These objects are high-order g-mode pulsators with periods between 8 to 80 hours (Kaye et al. 1999).

$\gamma$ Doradus stars are rather difficult seismic targets, for three main reasons: first, much fewer modes are actually observed than theory would predict. Second, the long pulsation periods of these stars require very extensive data sets taken with extremely stable instrumentation to detect low-amplitude pulsation modes reliably. Third, the pulsation periods are of the same order of magnitude as the stellar rotation periods, requiring a complicated theoretical treatment of rotation. However, seismology of these stars would still be highly interesting because their pulsation modes penetrate into the deep stellar interior, which usually is the region hardest accessible for seismic studies.

The solution to the problem may come from the fact that the $\gamma$ Doradus stars and the $\delta$ Scuti stars partly overlap in the HR diagram (Handler \& Shobbrook 2002). Consequently, there may be stars that have both sets of pulsation modes excited. Searches for such objects have, until recently, been unsuccessful, but Henry \& Fekel (2005) detected both kinds of pulsation in the single Am star HD 8801. An extended observational programme focused on this star appears quite worthwhile.

\subsection{Slowly Pulsating B stars}

Slowly Pulsating B (SPB) stars are main sequence stars with masses around $5 M_{\odot}$ that pulsate in high-order g-modes. The pulsation periods of these B3 - B9 stars are of the order of days, causing the same basic observational and theoretical difficulties as for the $\gamma$ Dor stars.

Nevertheless, some encouraging results for asteroseismology have already been obtained from the application of mode identification methods to SPB stars (De Cat et al. 2005). Therefore, some long-term monitoring of a group of selected SPB stars, with proper temporal sampling, appears to be a promising observational strategy to determine a sufficient number of pulsation frequencies and to provide reliable identifications of multiple modes for asteroseismic modelling. We note that the SPB stars overlap with the $\beta$ Cephei stars in the HR Diagram, and some stars showing both sets of pulsation modes may also be present (e.g., see Jerzykiewicz et al. 2005). 


\subsection{Beta Cephei stars}

The $\beta$ Cephei stars are singly- and multiperiodic radial and nonradial pulsators that oscillate in low-order $\mathrm{p}$ and $\mathrm{g}$ modes. The pulsation periods of these B0 - B2 main sequence stars (having masses of $\sim 9-17 M_{\odot}$, Stankov \& Handler 2005), are between $1.5-8$ hours.

Asteroseismology is already actively being done for these stars. The three independent modes of V836 Cen plus their rotational splitting allowed the inference of a small overshooting parameter $\left(\alpha_{o v}<0.15\right)$ and of the presence of differential interior rotation (Dupret et al. 2004). The four independent modes of $\nu$ Eri gave a similar result for $\alpha_{o v}(<0.12)$ and clear evidence for differential interior rotation with the convective core rotating about three times faster than the envelope (Pamyatnykh et al. 2004). It is possible that $\nu$ Eri is chemically stratified, with an increase of iron-group elements near the pulsational driving zone.

The reasons why mode identification and thus asteroseismology does work for the $\beta$ Cephei stars are that several of these stars have fairly high individual mode amplitudes (in excess of $0.01 \mathrm{mag}$ ) and that the underlying theory is comparably simple. In addition, all the $\beta$ Cephei stars seismically modelled so far have radial modes excited and identified. This is extremely helpful as it immediately sets tight constraints on the mean density of any possible seismic model. Therefore, the domain in the HR diagram to be searched for this model shrinks from a 2-D plane to three or less 1-D lines. We expect more seismic results for additional $\beta$ Cephei stars in the near future.

\subsection{Pulsating white dwarf stars}

There are three distinct groups of white dwarf pulsators that can be easily separated due to their spectral types (DO, DB and DA). All are g-mode pulsators with high radial overtones that result in periods between about 2 and 20 minutes. After the spectacular initial success of white dwarf asteroseismology mentioned before, few additional results could be obtained for some time. The reason is that few stars showed a pulsation spectrum sufficiently rich for seismic analyses.

However, in recent years the white dwarf interior models matured considerably, mostly aided by seismology of DB pulsators (Metcalfe 2005 and references therein). In particular, measurements of the ${ }^{12} \mathrm{C}(\alpha, \gamma){ }^{16} \mathrm{O}$ nuclear reaction rate could be obtained and be confronted with laboratory results. It is also possible that constraints on crystallisation of the interior of massive DA white dwarfs can be obtained (Brassard \& Fontaine 2005). Finally, the large number of recent new detections of pulsating white dwarf stars, mostly from the Sloan survey, should provide a number of interesting targets for future asteroseismic studies.

\subsection{Pulsating subdwarf B stars}

These pulsators are located on the extended horizontal branch in the HR diagram and have about half a solar mass. There are two subgroups: the EC 14026 stars oscillate with loworder $\mathrm{p}$ and $\mathrm{g}$ modes with periods of a few minutes, whereas the PG 1716 stars pulsate with high-order $g$ modes with periods around $1-2$ hours.

Seismic modelling of the interior structure of EC 14026 stars could not yet take place. The associated problems are familiar: often, much fewer modes are observed than predicted, and the stars may possibly have strong interior differential rotation (Kawaler \& Hostler 2005). Suggested nonradial m-mode splittings and the projected rotational velocities determined from spectroscopy do not always agree either. Some authors need to invoke modes of spherical degrees $\ell>2$ to explain the observed frequency spectra (e.g., Charpinet et al. 2005).

In conclusion, the application of mode identification methods is also desperately needed for the EC 14026 stars. So far, this was hampered by the extremely short periods combined with the low amplitudes of the pulsations. The solution may come from high-speed multichannel multicolour photometry (Jeffery et al. 2004). 
The PG 1716 stars appear to be even more difficult targets because of their longer periods and few modes so far detected. It is interesting to note that, very similar to the $\delta \mathrm{Sct} / \gamma$ Dor and $\beta$ Cep/SPB star pairs, "hybrid" short/long period sdB pulsators (Baran et al. 2005, Schuh et al. 2005) were also found. Again, such stars potentially provide a wealth of information on stellar interiors.

\section{Conclusions and outlook}

In our view, the future of asteroseismology is a bright one. Some classes of pulsating stars recently became available for seismic modelling. Mode identification methods are maturing and will become more and more reliable. Several interesting individual objects were discovered recently ("hybrid" pulsators showing both low-order $\mathrm{p}$ and $\mathrm{g}$ modes as well as high-order $\mathrm{g}$ modes). Because of the construction of giant mirrors, telescopes of the $2-4$ metre class are becoming more easily available for stellar astronomy, which will be particularly helpful for the application of spectroscopic mode identification methods. Present asteroseismic satellite missions (in particular MOST, Walker et al. 2003) are already yielding data of unprecedented quality, and it is hoped that future space projects (e.g., COROT, Baglin 2003) will open further dimensions in precision asteroseismology.

Acknowledgments. I am grateful to the organisers of the asteroseismology session at JENAM 2005 for inviting me to this inspiring conference.

\section{References}

Baglin, A. 2003, Advances in Space Research 31, 345

Balona, L. A. 2000, MNRAS 319, 606

Baran, A., et al. 2005, MNRAS 360, 737

Bedding, T. R., et al. 2004, ApJ 614, 380

Brassard, P., Fontaine, G. 2005, ApJ 622, 572

Breger, M., et al. 2005, A\&A 435, 955

Butler, R.P., et al. 2004, ApJ 600, L75

Charpinet, S., Fontaine, G., Brassard, P., Green, E.M., Chayer, P. 2005, A\&A 437, 575

Christensen-Dalsgaard, J. 2002, Rev. Mod. Phys. 74, 1073

Cunha, M. 2001, MNRAS 325, 373

Daszyńska-Daszkiewicz, J., Dziembowski, W. A., Pamyatnykh, A.A. 2003, A\&A 407, 999

Daszyńska-Daszkiewicz, J., Dziembowski, W.A., Pamyatnykh, A.A. 2005, Mem. Soc. Astr. Ital., in press (astro-ph/0509449)

De Cat, P., et al. 2005, A\&A 432, 1013

Dupret, M.A., et al. 2004, A\&A 415, 251

Dziembowski, W.A. 1977, Acta Astr. 27, 203

Handler, G., et al. 2000, MNRAS 318, 511

Handler, G., Shobbrook, R.R. 2002, MNRAS 333, 251

Hatzes, A.P. 1998, MNRAS 299, 403

Henry, G.W., Fekel, F.C. 2005, AJ 129, 2026

Jeffery, C.S., Dhillon, V.S., Marsh, T.R., Ramachandran, B. 2004, MNRAS 352, 699

Jerzykiewicz, M., et al. 2005, MNRAS 360, 619

Kawaler, S.D., Hostler, S.R. 2005, ApJ 621, 432

Kaye, A.B., et al. 1999, PASP 111, 840

Kurtz, D.W., Elkin, V.G., Mathys, G. 2005, MNRAS 358, L6

Kurtz, D.W., et al. 2005, MNRAS 358, 651

Ledoux, P. 1951, ApJ 114, 373

Metcalfe, T.S. 2005, MNRAS 363, L86

Pamyatnykh, A.A., Handler, G., Dziembowski W.A. 2004, MNRAS 350, 1022

Ryabchikova, T., Piskunov, N., Kochukhov, O., Tsymbal, V., Mittermayer, P., Weiss, W.W. 2002, A\&A 384,545 
Schuh, S., et al. 2005, in "Cool White Dwarfs from the SuperCOSMOS and Sloan Digital Sky Survey", eds. D. Koester, S. Moehler, ASP Conf. Ser. 334, 530

Stankov, A., Handler, G. 2005, ApJS 158, 193

Tassoul, M. 1980, ApJS 43, 469

Unno, W., Osaki, Y., Ando, H., Saio, H., Shibahashi, H. 1989, “Nonradial Oscillations of Stars”, University of Tokyo press, 2nd edition

Walker, G., et al. 2003, PASP 115, 1023

Winget, D.E., et al. 1991, ApJ 378, 326

Winget, D.E., et al. 1994, ApJ 430, 839 